\title{
Kheri (Acacia chundra, family: Mimosaceae) gum: Characterization using analytical, mathematical and pharmaceutical approaches
}

\author{
Rishabha Malviya ${ }^{1,2, A, B, D-F}$, Pramod Sharma ${ }^{1, D}$, Susheel Dubey ${ }^{3, F}$ \\ 1 Polymer Science Laboratory, Department of Pharmacy, School of Medical \& Allied Sciences, Galgotias University, Greator Noida U.P., India \\ 2 Department of Pharmacy, Uttarkhand Technical University, Dehradun, India \\ ${ }^{3}$ Siddarth Institute of Pharmacy, Dehradun, Uttarkhand, India \\ A - research concept and design; $\mathrm{B}$ - collection and/or assembly of data; $\mathrm{C}$ - data analysis and interpretation; \\ $D$ - writing the article; $E$ - critical revision of the article; $F$ - final approval of the article
}

Address for correspondence
Rishabha Malviya
E-mail: rishabhamalviya19@gmail.com
Funding sources
none declared
Conflict of interest
none declared
Acknowledgements
Authors are highly thankful to Prof. D. K. Chauhan from the
DD Pant Interdisciplinary Research Laboratory, Department
of Botany, University of Allahabad, India, for the authentication
of plant materials. Authors would like to thank the Indian
Institute of Technology, New Delhi, India, for carrying out mass
spectroscopy and NMR study.

Received on March 15, 2017

Reviewed on June 5, 2017

Accepted on August 20, 2017
DOI

10.17219/pim/76515

\section{Copyright}

๑) 2017 by Wroclaw Medical University

This is an article distributed under the terms of the Creative Commons Attribution Non-Commercial License (http://creativecommons.org/licenses/by-nc-nd/4.0/)

\begin{abstract}
Background. Natural polymers have been used in medical, pharmaceutical, cosmetic and food industry. They should be characterized before their possible applications in different industries.

Objectives. The objective of this study was to characterize Kheri (Acacia chundra, family: Mimosaceae) gum using analytical, mathematical and pharmaceutical approaches.

Material and methods. Crude Kheri gum (KG) was purified using distilled water as a solvent and ethanol as a precipitating agent. KG was characterized in terms of phytochemical screening, micromeritic properties, microbial load, ash value, rheological behavior, solid state ${ }^{1} \mathrm{H}$ nuclear magnetic resonance (NMR), mass spectra and Fourier-transform infrared spectroscopy (FTIR) studies for their possible applications in food, cosmetics and pharmaceutical industry.

Results. Studies show that KG contains carbohydrates, while protein, fat, volatile oils, alkaloids and glycosides are absent. $1 \%$ aqueous solution of polysaccharide showed $25.58 \times 10^{3} \mathrm{~kJ} / \mathrm{kg}$ activation energy and 1.39 Reynold's number. Viscosity average molecular weight of purified gum was found $1.73 \times 10^{5} \mathrm{D}$. Thermodynamic parameters, i.e., change in enthalpy $\Delta H v$ and change in enthalpy $\Delta H v$, were found to be $12.26 \times 10^{3} \mathrm{~kJ} / \mathrm{mol}$ and $24.47 \mathrm{~kJ} / \mathrm{mol}$, respectively. Mathematical approach also determined the rod shaped conformation of KG in aqueous solution. IR spectroscopic study shows the presence of free (COO-) and esterified (COO-R) carboxylic acid, ether ( $(-0$ stretching), galacturonic acid and mannose in polysaccharide ${ }^{1} H$ NMR study predicts presence of tetrahydropyran hydrogen in molecule. Furthermore, KG was also characterized as a suspending agent using paracetamol as a model drug. Flow rate, pH, particle size and settling behavior of suspensions were evaluated. Initial particle size of dispersed phase particles does not change significantly after 45 days.
\end{abstract}

Conclusions. From the findings of the research it can be concluded that $\mathrm{KG}$ can be used as an excipient in cosmaceuticals and pharmaceuticals and its characteristic rheological behavior may attract rheologists.

Key words: characterization, suspending agent, rheological behavior, Kheri gum, spectra 


\section{Introduction}

Water-soluble gums are also known as hydrocolloids. Hydrocolloids have been used in pharmaceutical, agriculture, food and cosmetic industry depending on their characteristics, rheological and molecular behavior. Gums have been successfully used for the treatment of industrial effluents. They are generally used as a gelling agent, a thickening agent, an emulsifying agent and a suspending agent due to their long, branched, chain structure. The chain length, ring structure and molecular weight determine the interaction to fluid, hence rheological behavior. ${ }^{1}$ Gums are obtained as exudates mainly from fruit and the trunk of plants after injury or incision, or after microbial invasion. Gum exudates are nodule or ribbon-shaped and act as a protective measure against a microbial attack. Gums are either a metabolic product of a plant or produced to conteract undesirable conditions.

Kheri gum (KG) (Acacia chundra, family: Mimosaceae) is widely distributed in the Gujarat, Maharashtra and Rajasthan regions of India. The trees have medicinal properties and are used as a source of timber and fodder. Gum obtained from the tree is a good substitute for acacia gum. ${ }^{2}$ The rheological behavior of the polymeric solution has been a topic of great interest among rheologists for their possible use in food, cosmetics and the pharmaceutical industry. Significant advances have been made in the characterization of the polymer, and in this paper green technology and mathematical approaches were used to characterize the rheological behavior of the polymer. The objective of the present research work was to characterize KG for their possible pharmaceutical and biomedical applications.

\section{Material and methods}

Crude gum was purchased from a local shop in New Delhi, India (Tyagi Herbal Products, Khari Bawali, Chadani Chowk). The gum was authenticated by Professor D. K. Chauhan (Department of Botany, University of Allahabad, India).

\section{Purification of polysaccharide}

Crude gum was dissolved in a sufficient amount of purified water and heated up to $40^{\circ} \mathrm{C}$. After $2 \mathrm{~h}$, the gum solution was filtered through a double folds muslin cloth to remove un-dissolved portion. The gum was precipitated by using ethyl alcohol and dried in an oven at $40^{\circ} \mathrm{C}$. Further, the gum was powdered, passed through 60\# $(0.25 \mathrm{~mm})$ sieve and stored in an airtight polypropylene jars under desiccated condition.

\section{Characterization of neutral ginseng polysaccharides}

\section{Phytochemical screening of gum}

As described by authors elsewhere, tests for carbohydrates, protein, fats, organic acids, glycosides, tannins and alkaloids were carried out for the phytochemical characterization of purified $\mathrm{KG}$. $^{3}$

\section{Organoleptic characteristics of gum}

Organoleptic properties are directly characterized using sense organs, which is why they are called organoleptic properties. Color, odor, taste, fracture and texture were evaluated to characterize the gum. ${ }^{4}$ A protocol was prepared to evaluate the taste and odor of KG. A total of 6 volunteers were selected for the study, with an age group of 18 to 35 years, out of which 3 were males and 3 females. Informed consent was obtained from volunteers. Volunteers were excluded if they had any of the following diseases or disorders:

- disease which affects taste perception, such as a thyroid disorder, diabetes mellitus and Cushing's syndrome, etc.;

- internal disorders, such as renal and hepatic disorders;

- middle ear infections;

- pregnancy, smoking behaviour, alcoholic, use of other medication, impaired perception towards taste and allergic reactions to artificial sweeteners.

To rate the taste of $\mathrm{KG} ; 0.32 \mathrm{M} \mathrm{NaCl}, 1 \mathrm{mM}$ quinine, $1 \mathrm{M}$ $\mathrm{NaCl}$ and $1 \% \mathrm{w} / \mathrm{v}$ solution of $\mathrm{KG}$ were painted over the tip of the tongue, sipped and swished in the mouth for $15 \mathrm{~s}$, and then spat out. Volunteers rated the intensity of the taste on GLMS. Standard numerical value of taste for $1 \mathrm{mM}$ quinine, $1 \mathrm{M} \mathrm{NaCl}$ and $0.32 \mathrm{M} \mathrm{NaCl}$ was 10,7 and 5 , respectively.

To determine the odor of KG, the smell of natural gas, smoke, orange and chocolate was used for identification. Volunteers who were not able to identify the odor were excluded from the study. Laboratory temperature was maintained at $25-27^{\circ} \mathrm{C}$ for the comfort of the volunteers. Odor intensity and intensity level were presented in Table 1.

\begin{tabular}{|c|l|l|}
\hline \multicolumn{2}{|c|}{ Table 1. Intensity level value for odor intensity } \\
\hline Scale number & Odor intensity & Intensity level \\
\hline 1 & extremely strong & A \\
2 & very strong & B \\
3 & strong & C \\
4 & distinct & D \\
5 & weak & E \\
6 & very weak & F \\
7 & not perceptive & G \\
\hline
\end{tabular}




\section{Solubility profile and $\mathrm{pH}$}

As described in a previous publication, 1 part of dry powdered polysaccharide was shaken with different solvents and the solubility was determined. One percent w/v solution of powdered gum was prepared and the $\mathrm{pH}$ was determined. ${ }^{4}$

\section{Particle size analysis}

Particle size of powdered gum sample was analyzed using optical microscopy - (Globus, Ph/L/16/02) (Rajas, Noida, Uttar Pradesh, India). Magnification value of microscope was calculated using overlapping point of eye piece and stage micrometer. In this measurement, 50 particles in 5 different fields were examined.

\section{Limit test for heavy metals}

Limit test for heavy metals $(\mathrm{Pb})$ and arsenic were carried out as per procedure mentioned in "Indian Pharmacopoeia" ${ }^{5}$

\section{Qualitative test for $\mathrm{Cl}^{-}$and $\mathrm{SO}_{4}^{2-}$}

Qualitative tests for $\mathrm{Cl}^{-}$and $\mathrm{SO}_{4}^{2-}$ were examined using the method given in "Indian Pharmacopoeia".

\section{Determination of microbial load}

Microbial load was determined to detect the presence of viable forms of microbes (bacteria, fungi and yeast). The method is based on the principle that in a nutritive medium, microbes grow and their presence can be detected by turbidity in the clear solution. Microbial load was determined as per Indian Pharmacopoeia. ${ }^{5}$ Values were shown as an average of triplicate studies with standard deviation.

Determination of total ash, total soluble ash, acid insoluble ash and sulphated ash

Total ash and related parameters have been used to characterize natural polymers. Standard AOAC method was used to estimate ash value of neutral ginseng polysaccharides (NGP). ${ }^{6}$ One gram of polysaccharide was taken and transferred to a pre-ignited and pre-weighed crucible and the total weight of the crucible with sample was noted. Further, the gum crucible was transferred into a furnace. The ignition temperature was maintained at $550^{\circ} \mathrm{C}$ for $24 \mathrm{~h}$. Ash value was calculated using equation 1. Afterwards, the recovered ash was used to calculate the total soluble ash using equation 2 :

$$
\text { total ash }(\%)=\frac{\text { actual weight of ash }}{\text { original weight of sample }} \times 100
$$

total soluble ash $=$ total ash (\%) - total insoluble ash (\%) (2).
Acid insoluble ash and sulphated ash were calculated after the ash was treated with $\mathrm{HCl}$ and $\mathrm{H}_{2} \mathrm{SO}_{4}$, respectively. Different ash values were shown as an average of triplicate studies with standard deviation.

\section{Viscosity measurement}

To determine viscosity $1 \% \mathrm{w} / \mathrm{v}$ solution of polysaccharide was prepared in purified water. Relative viscosity was determined using Ostwald's capillary viscometer (Rajas, Noida, Uttar Pradesh, India).

The effect of shear rates on the viscosity of the polymer was measured using Brookfield viscometer (National Analytical Corporation, Maharashtra, India). Viscosity of the polymer was measured at shear rates from 0.1 to $2.0 \mathrm{~s}^{-1}$ at $27^{\circ} \mathrm{C}$. Relative viscosity $\left(\eta_{r e l}\right)$, specific viscosity $\left(\eta_{s p}\right)$ and reduced viscosity $\left(\eta_{\text {red }}\right)$ was calculated using Equations 3, 4 and 5 at $27^{\circ} \mathrm{C}$, respectively:

$$
\begin{gathered}
\eta_{r e l}=\frac{\eta}{\eta_{0}} \\
\eta_{s p}=\eta_{r e l}^{-1} \\
\eta_{\text {red }}=\frac{\eta_{s p}}{C}
\end{gathered}
$$

where $\eta$ is intrinsic viscosity and $C$ is concentration of polymer.

\section{Effect of electrolytes and surfactant on the viscosity of polysaccharide}

The effect of electrolytes (calcium chloride, sodium chloride, and potassium chloride), surfactant (sodium lauryl sulphate) and citric acid on the viscosity of $1 \% \mathrm{KG}$ solution was determined. One percent solution of electrolytes, surfactant and citric acid were individually prepared and $5 \mathrm{~mL}$ of the prepared solutions were added to $100 \mathrm{~mL} 1 \%$ solution of KG. Then, the viscosities of individual samples were determined at $27^{\circ} \mathrm{C}$.

\section{Effect of temperature on the viscosity} and determination of thermodynamic parameters

Temperature changes the viscosity of liquid. The viscosity of liquid decreases with the increase in temperature. The effect of temperature on the viscosity was shown as a graph of viscosity vs the centigrade temperature.

The effect of temperature on the viscosity can be shown by the Arrhenius equation (equation 6):

$$
\eta=A \exp \frac{E}{R T}
$$

where $A$ is a constant for a liquid. Activation energy for viscous flow can be calculated from the slope of the graph 
between the logarithm of viscosity and reciprocal of the Kelvin temperature. Ideally, the plot between $\ln \eta$ and $1 / T$ should be linear and the value of the slope is equal to $E / R$.

Osborne Reynolds also describes an exponential equation to describe the effect of temperature on the viscosity as per equation 7 :

$$
\eta=R \exp [\alpha T]
$$

where $R$ is Reynolds number and $\alpha$ is a constant. Reynolds number and $\alpha$ was calculated from the graph between $\ln$ viscosity and temperature.

Thermodynamic parameters such as change in enthalpy $\Delta H v$ and change in entropy $\Delta S v$ of polymeric solution can be calculated using Frenkel-Eyring equation 8:

$$
\ln \left[\frac{\eta}{T}\right]=\left(\ln A-\frac{\Delta S v}{R}\right)+\frac{\Delta H v}{R T}
$$

From equation 8 , the graph can be plotted between $\ln [\eta / T]$ and $1 / T$ having slope and intercept $\Delta H v / R$ and $\ln A-\Delta S v$ respectively. ${ }^{7}$

\section{Determination of intrinsic viscosity}

Hydrodynamic volume occupied by a single polymer molecule is measured in terms of intrinsic viscosity. Hydrodynamic volume depends upon the size and conformation of the polymeric chain. Generally, intrinsic viscosity is determined at a low polymer concentration. Intrinsic viscosity is measured by calculating specific viscosity at various concentrations at a fixed temperature and shear rate. Intrinsic viscosity can be determined using the Huggins equation (equation 9):

$$
\frac{\eta_{s p}}{C}=[\eta]+K[\eta]^{2} C
$$

$\eta_{s p} / C$ is known as reduced viscosity. It is clear from equation 8 that a graph between reduced viscosity and concentration should be linear with the slope showing the value of $K[\eta]^{2}$ and intercept $[\eta]$. Huggins constant $K$ can be calculated from the value of slope and $[\eta]$.

Kraemer equation (equation 10) can be used to analyze intrinsic viscosity:

$$
\ln \left(\frac{\eta_{s p}}{T}\right)=[\eta]+K_{0}[\eta]^{2} C
$$

where $K_{0}$ is Kraemer constant. A graph between $\ln \left(\eta_{s p} / C\right)$ and concentration $C$ should be a straight line with the slope showing the value of $K_{0}[\eta]^{2}$ and intercept [ $\left.\eta\right]$. Huggins constant $K_{0}$ can be calculated from the value of the slope and [ $\left.\eta\right]$.

Tanglertpaibul and Rao derived 3 equations (equations 11,12 and 13) for the determination of the intrinsic viscosity of a polymeric solution ${ }^{8}$ :

$$
\eta_{r e l}=1+[\eta] C
$$

$[\eta]$ is the slope of the graph plotted between $\eta_{r e l}$ and $C$.

$$
\eta_{r e l}=\mathrm{e}^{[\eta] C}
$$

$[\eta]$ is the slope of the graph plotted between $\ln \eta_{\text {rel }}$ and $C$.

$$
\eta_{\text {rel }}=1 /(1-[\eta] C)
$$

$[\eta]$ is the slope of the graph plotted between $1 /\left(1-\eta_{\text {rel }}\right)$ and $C$.

Molecular conformation, polymer interaction and coil overlap parameter

Generally, power law equation 14 is used to study the molecular conformation, polymer interaction and coil overlap parameter:

$$
\eta_{s p}={ }_{a} C^{b}
$$

Mathematically, equation 14 can be shown as equation 15 :

$$
\ln \eta_{s p}=\ln a+b \ln C
$$

The value of constant $b$ is important to determine the conformational behavior of a polymer in dilute solutions.

\section{Determination of molecular weight}

Concentration dependency of viscosity can be represented by equation 8 , where $K$ is known as Huggins constant. It was found that when the polymer is dispersed in a good solvent, the value of $K$ is near 0.35 and relatively more value is observed when the polymer is dispersed in a poor solvent. Constant $\alpha$ is known as "shape parameter" and is related to the conformation of the polymer in a particular solvent. The value of $\alpha$ is 0 for a nonsolvent (in which the polymer precipitates). In a good solvent, the value of $\alpha$ varies from 0.5 to 1 . Viscosityaverage molecular weight $(M)$ can be estimated from intrinsic viscosity using Mark Houwink equation (equation 16):

$$
[\eta]=k M^{\alpha}
$$

where $\alpha=0.732$ and $k=3.8 \times 10^{-4}$, where 2 constants are characteristics of each solute-solvent system. For most of the polysaccharides, the value of $M$ shows a weight average value, in spite of the number average value. ${ }^{9}$

\section{Determination of surface tension}

One percent w/v solution of the gum was prepared using distilled water, and surface tension was determined using stalagmometer (Rajas, Noida, Uttar Pradesh, India). Surface tension was shown as the average of triplicate studies with standard deviation. 


\section{Foaming capacity and foam stability}

To measure foaming capacity, $1 \mathrm{~g}$ of polysaccharide was mixed with $100 \mathrm{~mL}$ of distilled water (q.s.). The gum was dissolved using a high-speed blender (at $5000 \mathrm{rpm}$ ) and the prepared solution was immediately transferred into a $250 \mathrm{~mL}$ measuring cylinder. Foaming capacity (\%) and foam stability (\%) were calculated using equation 17 and equation 18 , respectively. Foam stability was measured after $15 \mathrm{~min}$. Foaming capacity and foam stability was determined 3 times and the results were shown as the average of triplicate studies with standard deviation.

$$
\begin{gathered}
\text { foam capacity }(\%)=\frac{\text { foam volume after blending }- \text { foam volume before blending }}{\text { foam volume before blending }} \times 100 \\
\text { foam capacity }(\%)=\frac{\text { foam volume after time }(t)}{\text { initial foam volume }} \times 100
\end{gathered}
$$

\section{Swelling index and water holding capacity}

As described in a previous publication, swelling index (\%) of the polymer was calculated. To measure water holding capacity, 1 gm of polysaccharide was added in $25 \mathrm{~mL}$ of distilled water and kept for $12 \mathrm{~h}$, which was followed by centrifugation at $5000 \times \mathrm{g}$ for $30 \mathrm{~min} .{ }^{3}$ Supernatant was removed and the water-holding capacity was calculated using equation 19 . The results were shown as an average of triplicate studies with standard deviation.

$$
\text { water holding capacity }(\%)=\frac{\text { weigh of wet sample }- \text { weight of dry sample }}{\text { weight of dry sample }}
$$

\section{Micrometric properties and flow behavior of polymer}

Micrometric properties such as bulk density, tapped density, bulkiness, Carr's Index, Hausner's ratio and angle of repose of gum was performed 3 times as described in previous publications. ${ }^{3}$

\section{Surface morphology}

Surface morphology of powdered gum was studied using scanning electron microscopy (SEM). SEM study was performed at the Department of Textile Engineering, Indian Institute of Technology New Delhi, India.

\section{Fourier transform infrared spectroscopy}

Fourier-transform infrared spectroscopy (FTIR) analysis was performed at Central Instrument Facilities, School of Medical and Allied Sciences, Galgotias University, Greater Noida, India. Dried powdered gum sample was put on the analyzer plate of Bruker ATR equipment (Alpha, ECD-ATR) (Bruker Optics K.K. Yokohama, Japan). The obtained spectra was recorded and interpreted to analyze functional groups present in the polysaccharide.

\section{Nuclear magnetic resonance (' $\mathrm{H}$ NMR)}

Nuclear magnetic resonance (NMR) spectra were recorded using dimethyl sulfoxide (DMSO) as a solvent at the Indian Institute of Technology, New Delhi, India.

\section{Mass spectroscopy study}

Mass spectrometry is an important tool used to analyze the structure of a polymer. Mass spectra's are used to elucidate the structural characterization with linkage, bond type and configuration of polysaccharides. Mass spectral study was performed at the Indian Institute of Technology, New Delhi, India, using matrix assisted laser desorption/tonization - time of flight (MALDI-TOF) mass spectroscopy.

\section{Formulation of suspension}

Paracetamol suspensions of NGP were prepared and evaluated for possible suspending properties of polysaccharide. In this study, NGP $(0.5,0.75,1.25,1.5$ and $1.75 \% \mathrm{w} / \mathrm{v}$ ) was used as a suspending agent, benzoic acid (1\%) as preservative and paracetamol as model drug. Suspensions were prepared by triturating gum and drug simultaneously, followed by the addition of preservatives.

\section{Organoleptic characteristics and $\mathrm{pH}$ of suspension}

Organoleptic properties, such as color, odor and taste of prepared formulations, were analyzed by direct perception. Organoleptic properties are characteristics that can be observed by sense organs. Eyes were used to observe color while nasal cavity to detect odor. The taste of formulations was observed by taking $0.1 \mathrm{~mL}$ of the formulation on the tongue as determined for KG. 
The $\mathrm{pH}$ of the formulations was measured by a laboratory pH meter - Decibel Digital Technologies, DB-1002 (Philips India Limited, Chandigarh, India).

\section{Rheological characteristics of suspension}

The time required for each suspension sample to flow through a $10 \mathrm{~mL}$ pipette was determined and the apparent viscosity was calculated using equation 20 :

$$
\text { flow rate }=\frac{\text { volume of pipette }[\mathrm{mL}]}{\text { flow time }[\mathrm{s}]}
$$

Viscosity of samples was also determined at $27^{\circ} \mathrm{C}$ using Brookfield viscometer at 25 RPM. ${ }^{10}$

\section{Settling behavior of suspension}

Prepared suspensions were transferred into a graduated cylinder and the sediment volume was observed after a predetermined time (at every $1 \mathrm{~h}$ for $7 \mathrm{~h}$ and then every $24 \mathrm{~h}$ for 7 days). The rate of settling (F) was calculated using equation 21:

$$
F=\frac{V_{u}}{V_{o}}
$$

where $V_{u}$ is the volume of sediment and $V_{o}$ is the total volume of suspension.

Redispersion time of the prepared formulation was measured (after 45 days) by inverting the formulation and additional required time for uniform distribution of disperse phase was determined.

Particle size analysis was also carried out for dispersed phase to study any crystal growth or particles aggregation.

\section{Results and discussion}

The content of any polysaccharide may be changed significantly, even in small variations of environmental condition and soil characteristics. After the study was conducted, crude gum sample was stored as a reference sample for future prospectives. Thin-layer chromatography (TLC) report, infrared (IR) spectra, Mass spectra and NMR report was kept with sample for referencing purpose. In the future, any researcher would be able to differentiate their own KG from the studied KG based on reference data. On performing chemical test, it was found that carbohydrate was present in KG, while fat, tannin, glycoside, alkaloids, volatile oil, proteins and organic acids were absent. The gum was whitish brown, odorless and characteristic in taste, with rough fracture and irregular texture.

All the volunteers were able to rate the brightness of light, so no one was excluded from the study. On GLMS scale, the value of taste for KG can be denoted as " 0 ".

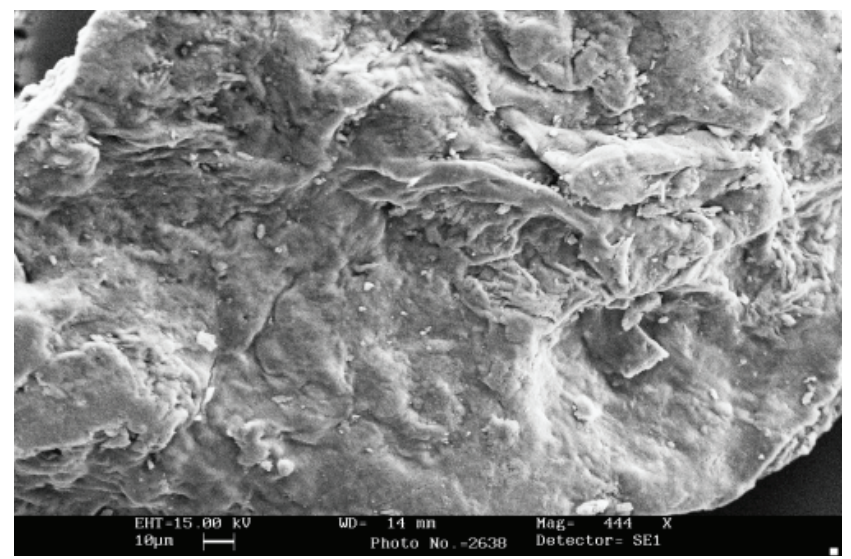

Fig. 1. SEM photograph of Kheri gum polysaccharide

All the volunteers were able to identify the odor of natural gas, smoke, orange and chocolate, so no one was excluded from the study. On the intensity scale, the intensity level of KG can be marked as "G".

Powdered gum was found to be soluble in both cool water $\left(25^{\circ} \mathrm{C}\right)$ as well as warm water $\left(40^{\circ} \mathrm{C}\right)$. Polysaccharide was insoluble in benzene, n-hexane, diethyl ether, chloroform, cyclohexane, acetone, ethanol and methanol. Kheri gum can be used as pharmaceutical excipient in dermal as well as oral formulation without any irritant effect as the $\mathrm{pH}$ of $1 \%$ of the solution was found to be $6.7 \pm 0.0 .67$. This shows the neutral nature of the polymer. The particles size of the powdered gum was ranged from 41.32 to $77.13 \mu \mathrm{m}$ with mean size of $61.2 \mu \mathrm{m}$.

Analysis determined that heavy metals (lead and arsenic) and $\mathrm{Cl}^{-}$and $\mathrm{SO}_{4}^{2-}$ were absent in powdered gum. The value of microbial load was found within in the pharmacopoeial limit. It was established at $83 \pm 10.11 \mathrm{CFU} / \mathrm{g}$ and $104 \pm 7.67 \mathrm{CFU} / \mathrm{g}$ for bacteria and fungi, respectively. This might be due to the use of alcohol during the precipitation process.

Total ash, water soluble ash, acid insoluble ash and sulphated ash of polysaccharide was found to be $12.5 \pm 0.13 \%$, $7.23 \pm 0.18 \%, 12.44 \pm 0.21 \%$ and $11.5 \pm 0.33 \%$, respectively. The value of ash content indicates the proportion of insoluble materials present in gum.

Viscosities of calcium chloride, sodium chloride, potassium chloride, sodium lauryl sulphate and citric acid containing solutions were found to be 1.055 ; 0.798; 0.790; 0.814 and 0.92 , respectively. The results showed that calcium chloride containing solution showed a relatively high viscosity, which may be due to the presence of divalent $\mathrm{Ca}^{2+}$ ions that further causes gel formation with polysaccharide. Sodium ions and potassium ions also form monovalent salts with polysaccharide. The solubility of potassium salts are higher than the sodium salts of polysaccharide, which is why potassium salts containing solutions showed less viscosity than sodium containing salts. In the presence of citric acid, the solubility of the polymer decreases and shows a relatively higher viscosity as compared to sodium and potassium salts containing solutions. 


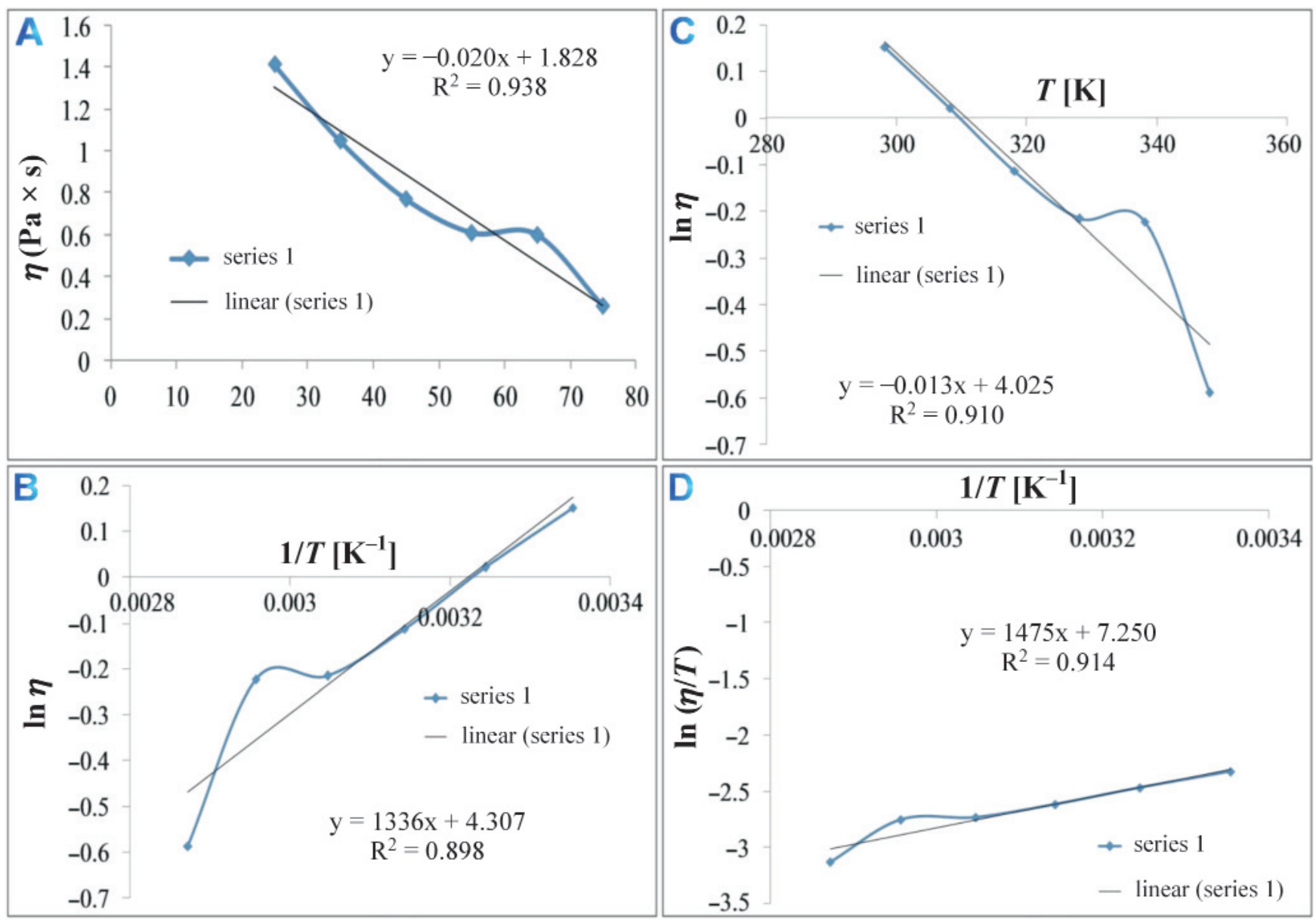

Fig. 2. A - effect of temperature on viscosity; B - determination of activation energy using Arrhenius equation (6); C - effect of temperature on the viscosity as per Reynold's equation (7); D - determination of thermodynamic parameters using Frenkel-Eyring equation (8)

To determine the effect of temperature on viscosity, a Brookfield viscometer was used. Viscosity is the measure of how easily molecules flow with respect to another molecule. It can be concluded from the Fig. $2 \mathrm{~A}$ that as the temperature increases, viscosity of polymeric solution decreases. Temperature increases the kinetic energy of molecules and thus promotes the easiness of flow. During literature survey, it was found that the plot of the logarithm of viscosity, $\ln (\eta)$, against the reciprocal of absolute temperature $(1 / T)$ for solutions is almost linear and activation energy can be calculated from the slope of the plot. This process is known as non-isothermal kinetic model for determination of activation energy. In case of non-linear plot, activation energy can be calculated for 2 continuous temperatures.

Activation energy can be estimated from the slope (equation 22) of Arrhenius equation (equation 6):

$$
\text { slope }=\frac{E}{2.303 R}
$$

where $R$ is gas constant and value of $R$ is $8.314 \mathrm{~kJ} \mathrm{~kg}^{-1} \mathrm{~mol}^{-1} \mathrm{~K}^{-1}$. Activation energy $E$ for $1 \% \mathrm{w} / \mathrm{v}$ solution of the polymer at $27^{\circ} \mathrm{C}$ was found to be $25.58 \times 10^{3} \mathrm{~kJ} \mathrm{~kg}^{-1}$.

This energy predicts polymer solutions sensitivity towards temperature. The value of $E$ is considered as an energy barrier for the gelation of the polymeric solution at a particular temperature. ${ }^{11}$ The intercept of the graph (Fig. 2B) was used to calculate Arrhenius constant and it was found to be 4.31 . The value of linearity $(0.898)$ for semilog plot of viscosity with the inverse of absolute temperature predicts the linear dependency of activation energy with different temperatures. Activation energy for a polymeric solution depends upon hydrogen bonding, configurational changes and hydrophobic interactions.

The effect of temperature on the viscosity of a polymer, as per Reynold's equation, was shown in Fig. 2C. A graph was used to calculate Reynold's number and constant $\alpha$. The value of Reynold's number and constant $\alpha$ was found to be 1.39 and 0.03 , respectively.

For the estimation of thermodynamic parameters of NGP solution, a graph was plotted based on FrenkelEyring equation (8) and shown in Fig. 2D. As per the Fig. 2D, the value of the slope was found to be 1475 and so a change in enthalpy $\Delta H v$ was $12.2610^{3} \mathrm{~kJ} / \mathrm{mol}$. In polymeric conformation, an energy barrier exists, which is measured in terms of the amount of energy required by molecules to jump from one equilibrium position to another. Mathematically, the energy barrier is measured by a change in enthalpy $\Delta H v$. 
The value of pre-exponential factor A was calculated by the intercept of the Fig. 1B based on Arrhenius equation ( $\ln A=-4.307)$. The value of pre-exponential factor $A$ was further used to calculate a change in entropy $\Delta S v$ using Frenkel-Eyring equation. The value of $\Delta S v$ was calculated and found to be $24.47 \mathrm{~kJ} / \mathrm{mol}$. The positive value of $\Delta S v$ indicated coiled conformation of the polymer in dilute solutions. ${ }^{12}$ The solubility of the polymer having coiled conformation can be improved by heating, which facilitates the unfolding of a polymer chain. Furthermore, after dissolution, the polymeric chains maintain coiled conformation, unless the chain is rigid. Positive value of $\Delta S v$ is due to a greater number of conformation of the polymer in dilute solution. Generally, entropy of mixing shows a lower value for dilute solutions and is governed by a very short ranged interactions, i.e. vander Walls force, hydrogen bonding and dipole-dipole. It was also found that the interaction between solvent-solvent, polymer-polymer and solventpolymer changes significantly during mixing of polymer due to conformational changes in polymeric chain.

Huggins, Kraemer and Tanglertpaibul and Rao techniques are classical methods for the estimation of intrinsic viscosity and are based on linear graphical extrapolation of the experimental data. ${ }^{13}$

Intrinsic viscosity was found to be $2.06 \mathrm{c}$-poise (Huggins equation) (Fig. 3A), 0.997 c-poise (Kraemer equation) (Fig. 3B), 0.623 cPoise (Tanglertpaibul and Rao equation 11), 0.176 cPpoise (Tanglertpaibul and Rao equation 12) and 2.618 c-poise (Tanglertpaibul and Rao equation 13).

Intrinsic viscosity depends upon molecular weight, conformational size and shape and specific volume. Interaction and conformation of polymer within solvent also depends upon concentration of polymer at fixed volume of solvent. Effective volume fraction of polymer in solution is expressed in terms of "space occupancy" or "coil overlap", which is a product of a concentration and intrin- sic viscosity ([$\eta] . c)$. As per equation 15, the plot between $\ln \eta_{s p}$ and $\ln C$ was found a linear slope and an intercept equal to $b$ and $l n$, respectively (not shown here). The value of $R^{2}$ was found to be 0.959 , and elicits good linearity. The value of $b$ was found to be 0.532 and is an important parameter for determining the conformation of polysaccharide. The value of constant $b$ is below 1 ; hence, it elicits the presence of rod like conformation of gum.

Viscosity average molecular weight of KG polysaccharide was found to be $1.73 \times 10^{5} \mathrm{D}$ using intrinsic viscosity data from Huggins equation 9. The surface tension of $1 \% \mathrm{w} / \mathrm{v}$ solutions of polysaccharide was found to be $136.43 \pm 3.13 \mathrm{dyn} / \mathrm{cm}^{2}$. Wetting and spreading properties was determined by the surface tension of any polymer. The surface tension also influences the effect of biological fluid on the pharmaceutical formulation made up of a respective polymer. The lower surface tension promotes better interaction and penetration of biological fluid within delivery system.

Foaming ability is an important parameter characterizing gum. Foam capacity and foam stability of $1 \% \mathrm{w} / \mathrm{v}$ solution of $\mathrm{KG}$ was found to be $58 \pm 1.37 \%$ and $63 \pm 1.09 \%$, respectively. Foam is generally produced by the turbulent flow, which causes the entrapment of air bubbles in liquid. Stability of foam depends upon the difference in density between the gaseous phase and the liquid medium. The use of surfactant decreases surface energy between 2 phases and improves the stability of the foam system. Polysaccharides are not used to prepare foam, but their presence improves the stability of other foams due to their 3-dimensional polymeric structure.

The swelling index of polysaccharide was found to be $70 \pm 4.33 \%$, while the water-holding capacity of the polymer was $95 \pm 5.67 \mathrm{~g}$ water/g of dry powdered polysaccharide. The value obtained after the study elicits the fact that the polymer has a good attraction towards water, so KG is hydrophilic in nature. The bulk density and tapped

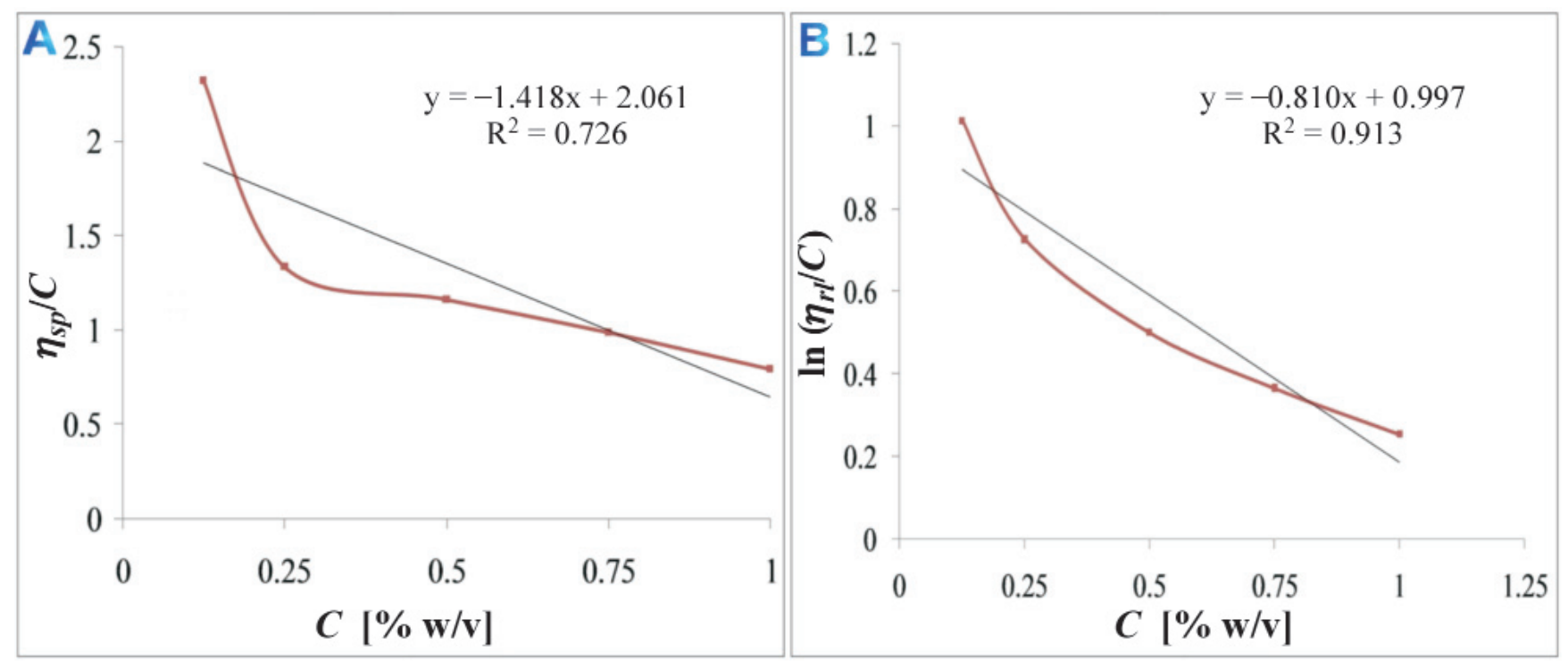

Fig. 3. Determination of intrinsic viscosity (A) using Huggins equation (9) and (B) Kraemer equation (10) 
density are used to characterize compactation properties and packing arrangements of polymer. KG was characterized for its micromertitic properties such as bulk density $(0.71 \pm 0.16 \mathrm{~g} / \mathrm{mL})$, tapped density $(0.83 \pm 0.04 \mathrm{mg} / \mathrm{mL})$, Carr's index (14.28 \pm 0.19$)$, Hausner's ratio $(1.16 \pm 0.07)$ and angle of repose $\left(19.8 \pm 0.53^{\circ}\right)$. The bulkiness value is used to describe 'heaviness' of powder and was $1.60 \pm 0.04$ $\mathrm{mL} / \mathrm{mg}$. The angle of repose is used to characterize the flow behavior of the polymer for better industrial applications. In the present study, the value of the angle of repose was $19.8 \pm 0.53^{\circ}$, which indicates "good" to "passable" flow behavior of KG. The value of tapped density was found to be more than the bulk density, which indicates the amount of entrapped air and packing arrangement of particles.

Infrared spectroscopy is used to determine the identity of the compound (Fig. 4). The region below wave number $1500 \mathrm{~cm}^{-1}$ shows much absorption caused as a result of bending and stretching vibrations. In this region, the numbers of bending vibrations are more than the number of stretching vibrations and are known as the finger print region. Stretching vibrations arise due to $\mathrm{C}-\mathrm{C}$, $\mathrm{C}-\mathrm{O}$ and $\mathrm{C}-\mathrm{N}$ bonds. The bonds in the region $1149-$ $1018 \mathrm{~cm}^{-1}$ were corresponding to $\mathrm{C}-\mathrm{O}-\mathrm{C}$ and $\mathrm{C}-\mathrm{O}-\mathrm{H}$ of glycosidic linkage. Absorption bands around 1618 and $1430 \mathrm{~cm}^{-1}$ were due to characteristic peaks of carboxylate group of galactoronic acid residue. Ethers show only one characteristics band in the region $1300-1050 \mathrm{~cm}^{-1}$. The peak at $1242.12 \mathrm{~cm}^{-1}$ shows $\mathrm{C}-\mathrm{O}-\mathrm{C}$ absorption spectrum of ether $\left(\mathrm{C}-\mathrm{O}\right.$ stretching). The peak at $1421.97 \mathrm{~cm}^{-1}$ represents the symmetric stretching of carboxylic group of uronic acid. Hence, uronic acid may be present in polysaccharide. The peak at $1620.15 \mathrm{~cm}^{-1}$ could be due to the stretching of mannose. The peak at $1742.78 \mathrm{~cm}^{-1}$ shows the characteristic peaks of $\mathrm{C}=\mathrm{O}$ stretching of saturated esters. The peak at $3561.66 \mathrm{~cm}^{-1}$ may arise due to intramolecular hydrogen bonded $\mathrm{O}-\mathrm{H}$ group. Carbonyl absorption bands showing the peak at 1620 and $1740 \mathrm{~cm}^{-1}$ were due to free (COO-) and esterified (COO-R) carboxyl groups, respectively.

Mass spectral analysis provides information about internal cleavage, sequence and branching. Interpretations of spectra provide knowledge about linkage side of monomer to form polymer. A mass spectrum of polysaccharide is shown in Fig. 5. Generally, carbohydrates show initial cleavage of glycosidic bond ( $-\mathrm{C}-\mathrm{O}-$ bond). Different literatures described fragmentation pattern through ring cleavages. After ring cleavage, both ions retain the charge of the molecular species. ${ }^{14}$

Nuclear magnetic resonance uses ${ }^{1} \mathrm{H},{ }^{13} \mathrm{C},{ }^{15} \mathrm{~N}$ and ${ }^{31} \mathrm{P}$ as tracer atoms for spatial information about the structure. Nuclear magnetic resonance study is widely used to elucidate the conformational aspects of polysaccharide structure. It was also found during literature survey that NMR study can be used to elicit the relationship between spatial structure of molecules and their biological activity. In this study, ${ }^{1} \mathrm{H}$ NMR spectra was used to characterize polysaccharide. Polysaccharide sample was analyzed and a spectrum was shown in Fig. 5, shift values were shown

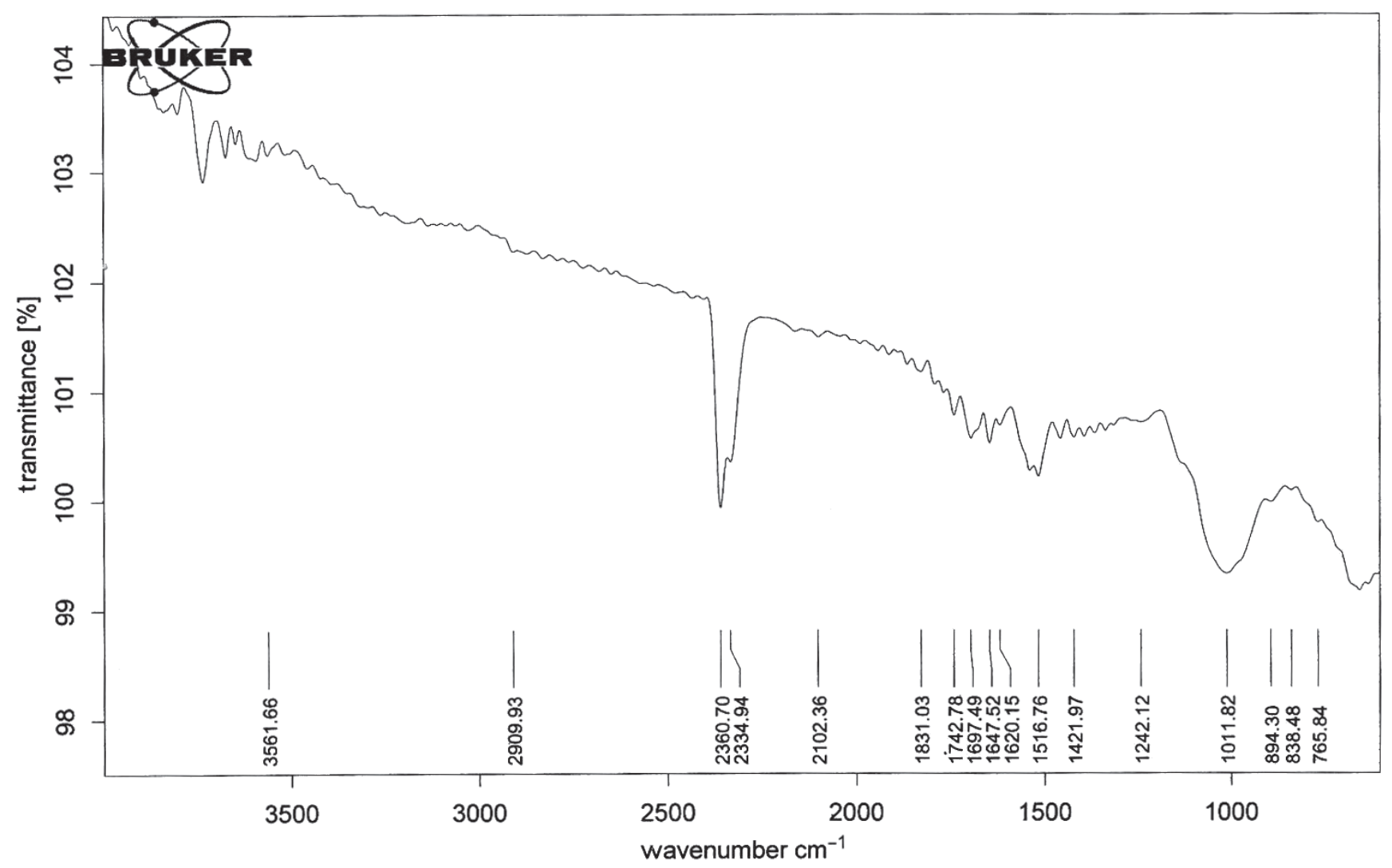

Fig. 4. Infrared spectra of Kheri gum 


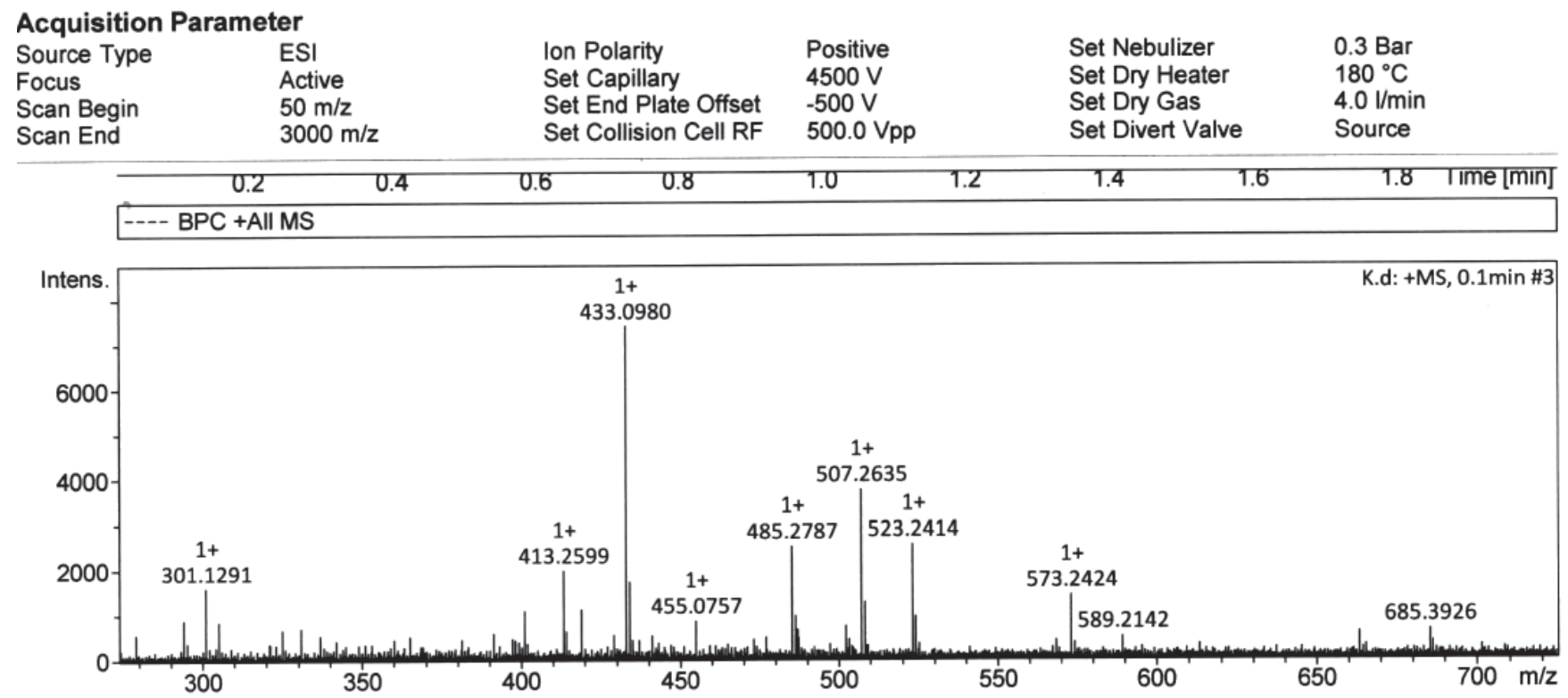

Fig. 5. Mass spectra of Kheri gum

relative to TMS. As shown in spectra (Fig. 6) shift value 5.417 PPM predicts the presence of tetrahydropyran hydrogen (1-alpha-O- from methane, 1-beta-O-C from methane), shift value 4.918 PPM shows the presence of tetrahydropyran hydrogen (1-alpha-O-C- from methane, 1-beta-C- from methane Tetrahydropyran) and the shift value of 3.39 PPM shows the presence of tetrahydropyran hydrogen (1-alpha-O-C- from methane, 1-beta-O-Cfrom methane, 1-beta-O- from methane). Crowded signals in the ${ }^{1} \mathrm{H}$ NMR spectra of KG are characteristic of polysaccharides and prove the presence of similar sugar residues.

Initially trail batches of suspensions were also prepared using relatively higher concentrations of NGP. It was observed during the study that suspension possesses higher sediment volume at higher concentrations of the polymer $(3-7 \% \mathrm{w} / \mathrm{v})$. It can be concluded from the high sediment volume that the polymer itself becomes a sediment at higher concentrations and it is an undesirable property for a suspension. Further, polymer concentration was reduced to prepare different batches of suspensions, viz. F1, F2, F3, F4 and F5.

Prepared suspensions were white-brown in color with a characteristic odor. The $\mathrm{pH}$ of the formulations was found $6.90 \pm 0.08$ to $6.96 \pm 0.06$. On GLMS scale, average value of taste for the prepared suspensions F1, F2, F3, F4 and F5 was found to be "7", "6", "6", " 5 " and " 5 " respectively.
For odor determination, the intensity scale was used and the intensity level of all the prepared suspensions was marked as "F".

Particle size analysis was carried out with an optical microscope. The effect of concentration of suspending agent (i.e., KG) on the $\mathrm{pH}$ and flow rate of suspension was shown in Table 2. It was analyzed from the result that the flow rate decreases as the concentration of KG increases. This is due to the 3-dimensional polymeric structure of the polymer and their more significant interaction at a higher concentration. A significant effect of the NGP on the redespersion time of suspension was observed due to the change in viscosity of suspension. The rate of settling the suspensions were evaluated for 45 days and the data shown in Table 3.

Results showed that sedimentation volume, particle size and redispersion time of the polymer are directly proportional to KG concentration, while reverse case is observed in the case of flow rate.

These studies used a cheap, biodegradable and effective excipient as a suspending agent in pharmaceutical suspensions. Interaction of the polymer with dispersed phase particles depends upon charge, conformation and size of the suspending agent in continuous phase. The suspending agent forms the bridge between the dispersed phase particles, because of the simultane-

Table 2. Characterization parameters of suspensions

\begin{tabular}{|l|c|c|c|c|c|}
\multirow{2}{*}{\multicolumn{1}{c}{ Parameters }} & \multicolumn{5}{c}{ Formulations } \\
\cline { 2 - 5 } & F1 & F2 & F3 & F4 \\
pH & $6.90 \pm 0.08$ & $6.92 \pm 0.07$ & $6.92 \pm 0.08$ & $6.93 \pm 0.06$ \\
Flow rate $[\mathrm{mL} / \mathrm{s}]$ & $4.33 \pm 0.00$ & $4.32 \pm 0.01$ & $4.32 \pm 0.00$ & $4.30 \pm 0.00$ & $1.43 \pm 16.00$ \\
Particle size $[\mu]$ & $1.29 \pm 17.03$ & $1.67 \pm 19.39$ & $1.33 \pm 23.26$ & $4.27 \pm 0.00$ \\
Redispersion time $[\mathrm{s}]$ & $39 \pm 3.47$ & $45 \pm 1.33$ & $46 \pm 2.67$ & $1.71 \pm 18.51$ & $53 \pm 2.56$ \\
\hline
\end{tabular}




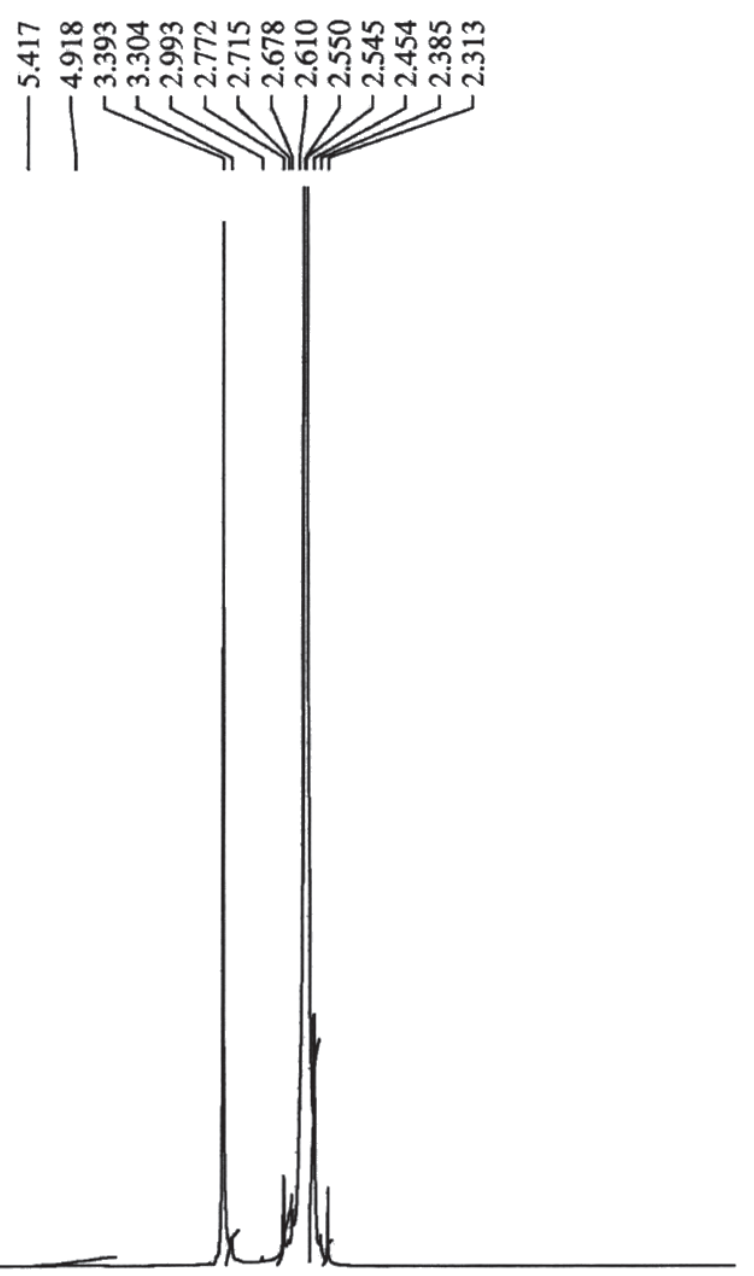

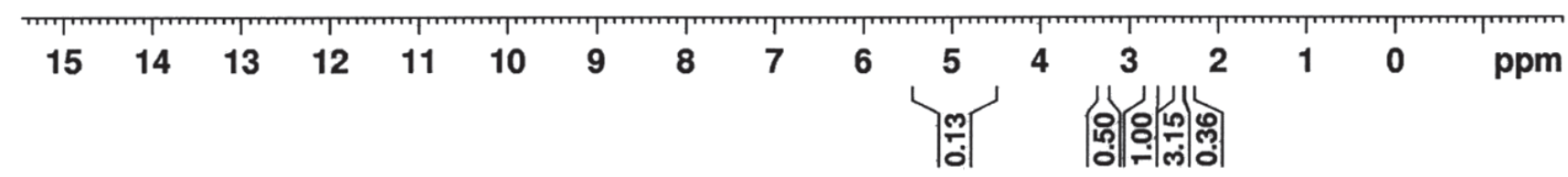

Fig. 6. ${ }^{1} \mathrm{H}$ NMR spectra of Kheri gum polysaccharide

ous adsorption of the suspending agent on the surface of suspended particles. At a low concentration of polysaccharide, less polymer was available to adsorb at the surface of suspended particles hence less number of particle-particle bridges were formed. In this condition the formation of flocculated suspension takes place. Intermediate concentration of KG leads to the formation of more particle-particle bridges and the forma- tion of flocculated suspension. A higher concentration of KG leads to the complete coverage of suspended particles and prevents close particle-particle interaction, leading to the formation of particle-particle bridges. In this condition, deflocculated suspension is formed. After 45 days, no crystal growth was analyzed by particle size analysis. It was observed that there is no significant change in the size of drug particles.

Table 3. Rate of settling of suspensions

\begin{tabular}{|c|c|c|c|c|c|c|c|c|}
\hline \multirow{2}{*}{ Formulation } & \multicolumn{8}{|c|}{ Sedimentation volume } \\
\hline & after 1 day & after 2 days & after 3 days & after 4 days & after 5 days & after 15 days & after 21 days & after 45 days \\
\hline F1 & 1 & 1 & 1 & 1 & 1 & 1 & 1 & 0.09 \\
\hline F2 & 1 & 1 & 1 & 1 & 1 & 1 & 1 & 0.15 \\
\hline F3 & 1 & 1 & 1 & 1 & 1 & 1 & 1 & 0.18 \\
\hline $\mathrm{F} 4$ & 1 & 1 & 1 & 1 & 1 & 1 & 1 & 0.30 \\
\hline F5 & 1 & 1 & 1 & 1 & 1 & 1 & 1 & 0.24 \\
\hline
\end{tabular}




\section{Conclusions}

It can be concluded from the findings that:

- KG can be effectively purified using water as a solvent and ethyl alcohol as a precipitating agent.

- KG gum shows the presence of carbohydrate, while fats and volatile oils are absent in polysaccharide.

- Positive value of change in entropy shows a higher number of conformation in dilute solution.

- Dilute solution of KG in water shows rod shaped conformation of polymer.

- IR spectroscopic study shows the presence of free (COO-) and esterified (COO-R) carboxylic acid, ether ( $\mathrm{C}-\mathrm{O}$ stretching), galacturonic acid and mannose in polysaccharide.

- ${ }^{1} \mathrm{H}$ NMR study predicts the presence of tetrahydropyran hydrogen in the molecule.

- Research also provides insights for the use of KG as a suspending agent, because prepared suspensions did not exhibit any significant change in particle size, $\mathrm{pH}$ and flow rate during storage.

\section{References}

1. Priscilla BSA, Luana CBBC, José AT, Carneiro-da-Cunha MG. Approaches in biotechnological applications of natural polymers. AIMS Mol Sci. 2016;3(3):386-425.

2. Malviya R, Sharma PK, Dubey SK. Antioxidant potential and emulsifying properties of Kheri (Acacia chundra, Mimosaceae) gum polysaccharide. Marmara Pharm J. 2017 [In press].

3. Pant S, Malviya R, Sharma P. Extraction and characterization of Boswellia serrata gum as pharmaceutical excipient. Polim Med. 2015;45(1):25-30.

4. Malviya R. Extraction characterization and evaluation of selected muclilage as pharmaceutical excipient. Polim Med. 2011;41(3):39-44.

5. Indian Pharmacopoeia. $7^{\text {th }}$ ed. New Delhi, India: Government of India, Controller of Publications; 2014:vol. 2.

6. AOAC, 2000. Official methods of analysis. Washington, USA: Association of official analytical chemist.

7. Eddy NO, Udofia I, Uzairu A, Odiongenyi AO, Obadimu C. Physicochemical, spectroscopic and rheological studies on Eucalyptus citriodora (EC) gum. J Polym Biopolym Phys Chem. 2014;2(1):12-24.

8. Vahid S, Hossein J, Mohammad SY. A comparison of various models for obtaining the intrinsic viscosity of salep gum and sweeteners mixture in dilute solutions. Int Food Res J. 2011;18(4):1457-1462.

9. Faria S, de Oliveira Petkowicz CL, Lemos de Moraisc SA, et al. Characterization of xanthan gum produced from sugar cane broth. Carbohydr Polym. 2011;88:469-476.

10. Higiro J, Herald T, Alavi S. Rheological study of xanthan and locust bean gum interaction in dilute solution. Food Res Int. 2006;39(2):165-175.

11. Nair SV, Oommen Z, Thomas S. Melt elasticity and flow activation energy of nylon 6/polystyrene blends. Mater Lett. 2002;57(2):475-480.

12. Morris ER, Cutler AN, Ross-Murphy SB, Rees DA. Concentration and shear rate dependence of viscosity in random coil polysaccharide solution. Carbohydr Polym. 1995;1(1):5-21.

13. Arvidson SA, Rinehart BT, Gadala-Maria F. Concentration regimes of solution of levan polysaccharide from Bacillus sp. Carbohydr Polym. 2006;65:144-149.

14. Spengler B, Dolce JW, Cotter RJ. Infrared laser desorption mass spectrometry of oligosaccharides: Fragmentation mechanisms and isomer analysis. J Am Chem Soc. 1990;62(17):1731-1737. 